Supporting Information for

\title{
Synergistic Effect of Binary Surfactant Mixtures in Two-phase and Three-phase Systems
}

\author{
Agata Wiertel-Pochopien ${ }^{1 *}$, Piotr Batys ${ }^{1}$, Jan Zawala ${ }^{1 *}$, Przemyslaw B. Kowalczuk ${ }^{2,3}$ \\ 1 Jerzy Haber Institute of Catalysis and Surface Chemistry, Polish Academy of Sciences, \\ Niezapominajek 8, 30-239 Krakow, Poland \\ ${ }^{2}$ Norwegian University of Science and Technology, Department of Geoscience and Petroleum, \\ S. P. Andersens veg 15a, 7031 Trondheim \\ 3 Wroclaw University of Science and Technology, Faculty of Chemistry, Wybrzeze \\ Wyspianskiego 27, 50-370 Wroclaw, Poland
}

*Corresponding authors: jan.zawala@ikifp.edu.pl; agata.wiertel-pochopien@ikifp.edu.pl 

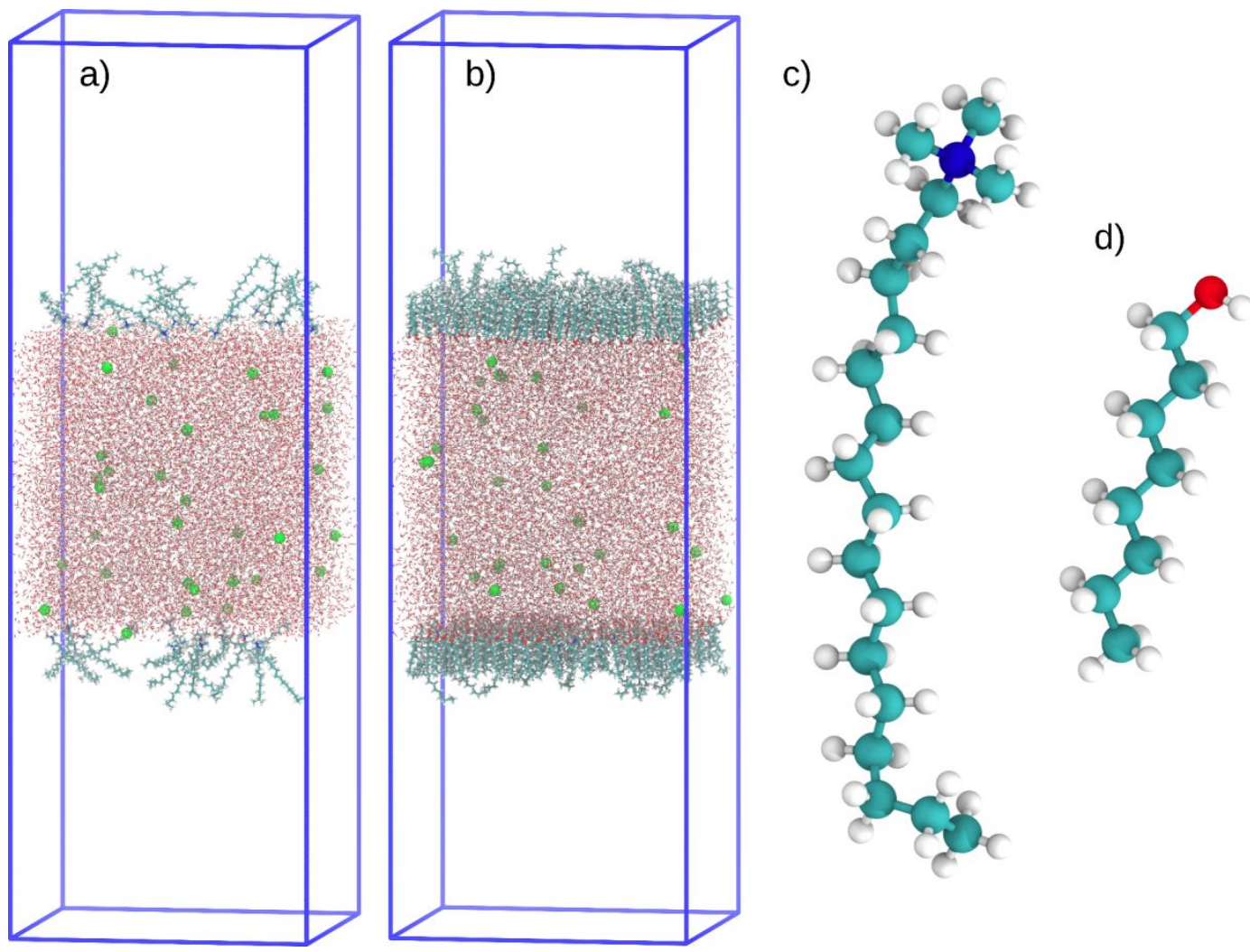

Figure S1. Snapshots showing initial configurations of CTAB only (a) and CTAB/OcOH mixture (b), as well as the molecular structure of $\mathrm{CTAB}(\mathrm{c})$ and $\mathrm{OcOH}(\mathrm{d})$.
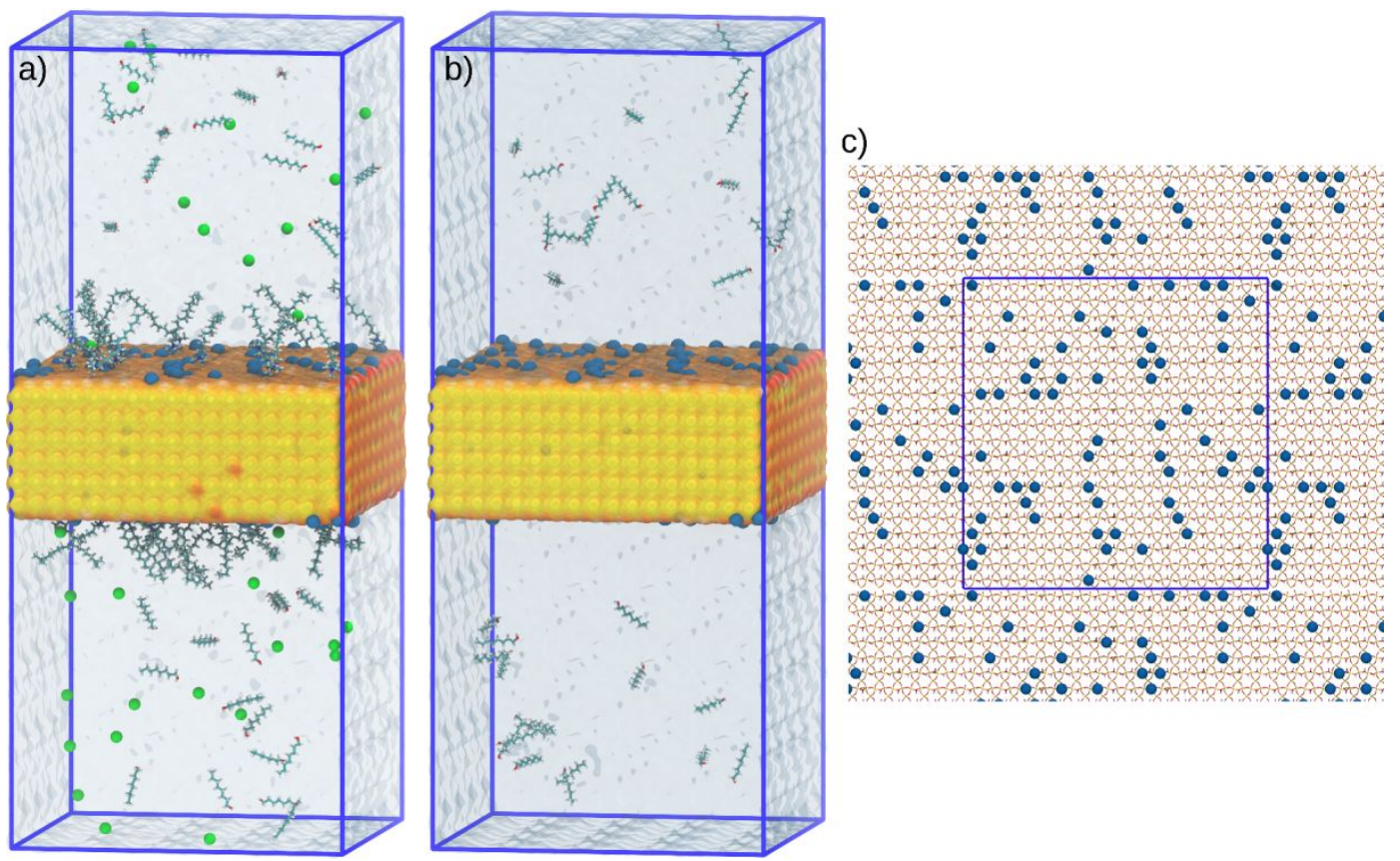

Figure S2. Snapshots showing initial configurations of $\mathrm{OcOH}$ solution (a) and $\mathrm{OcOH} / \mathrm{CTAB}$ mixture (b) at (001) quartz/water interface. (c) The initial distribution of $\mathrm{Na}^{+}$ions on (001) quartz. For the clarity, water is presented using surface representation. 

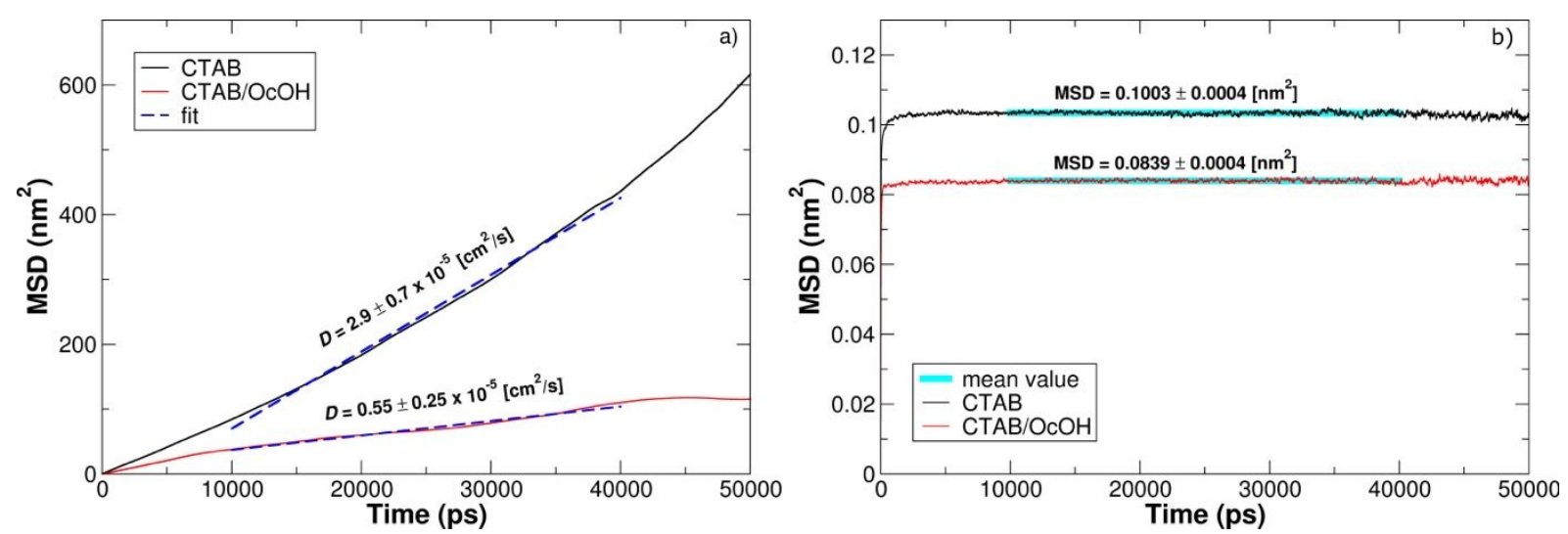

Figure S3. Mean squared displacement (MSD) in the $x y$ plane (a) and the $z$ direction (b). The diffusion coefficients $D$ were determined using the Einstein relation, within the range from 10000 to 40000 [ps]. To exclude the effect of the whole system fluctuation in $x y z$ directions, the center of mass motion was removed. 